\title{
Surface heterogeneity of 2005 UD from photometric observations
}

\author{
D. Kinoshita ${ }^{1}$, K. Ohtsuka ${ }^{2}$, T. Sekiguchi ${ }^{3}$, J. Watanabe ${ }^{3}$, T. Ito ${ }^{3}$, H. Arakida ${ }^{4}$, \\ T. Kasuga ${ }^{3}$, S. Miyasaka ${ }^{5}$, R. Nakamura ${ }^{6}$, and H.-C. Lin $^{1}$ \\ ${ }^{1}$ Institute of Astronomy, National Central University, 300 Jhongda Rd., Jhongli, Taoyuan, 32001, Taiwan \\ e-mail: kinoshita@astro.ncu.edu.tw \\ 2 Tokyo Meteor Network, 1-27-5 Daisawa, Setagaya, Tokyo, 155-0032, Japan \\ 3 National Astronomical Observatory of Japan, 2-21-1 Osawa, Mitaka, Tokyo, 181-8588, Japan \\ 4 Waseda University, 1-6-1 Nishi-Waseda, Shinjuku, Tokyo, 169-8050, Japan \\ 5 Tokyo Metropolitan Government, 2-8-1 Nishi-Shinjuku, Shinjuku, Tokyo, 163-8001, Japan \\ ${ }^{6}$ Grid Technology Research Center, National Institute of Advanced Industrial Science and Technology, Central 2, 1-1-1 Umezono, \\ Tsukuba, Ibaraki, 305-8568, Japan
}

Received 20 August 2006 / accepted 28 November 2006

\section{ABSTRACT}

\begin{abstract}
Context. The recently discovered Apollo-type near-Earth asteroid 2005 UD has been suggested to be a fragment of (3200) Phaethon. Aims. To test this hypothesis, we carried out photometric observations of 2005 UD using the 1-m telescope at Lulin Observatory. Methods. Multi-color photometry was used to compare the surface properties of (3200) Phaethon and 2005 UD. Surface-color variation due to the rotation was also examined.

Results. The time-resolved differential photometry showed clear brightness variation, and the lightcurve was fitted with a rotation period of $5.23 \mathrm{~h}$ and an amplitude of $0.44 \mathrm{mag}$. Using this rotational lightcurve, we derived the surface colors of 2005 UD. The surface of 2005 UD exhibits colors similar to those of F- and B-type asteroids, which is consistent with (3200) Phaethon. Furthermore, the $(R-I)$ color of 2005 UD shows variation during the rotation of the body.

Conclusions. The similarity of surface colors between (3200) Phaethon and 2005 UD observationally supports the hypothesis that 2005 UD is likely to be a fragment of (3200) Phaethon. A simple explanation for the inhomogeneity of the surface is that we see the surface and subsurface of the precursor object. Another explanation is the topographical structure that such as a large crater causes on this heterogeneous surface.
\end{abstract}

Key words. minor planets, asteroids - comets: general - meteors, meteoroids - techniques: photometric

\section{Introduction}

An Apollo-type near-Earth asteroid, (3200) Phaethon $(=1983 \mathrm{~TB})$, was discovered by the InfraRed Astronomical Satellite (IRAS) in October 1983 (Green \& Kowal 1983). Although the appearance of (3200) Phaethon is asteroidal, its orbit is highly eccentric with $e=0.89$, like those of comets. In addition, (3200) Phaethon is a strong candidate for the Geminid meteor stream (Whipple 1983; Gustafson 1989; Williams \& Wu 1993), so it is regarded as one of the most likely dormant cometary nuclei, whose cometary activity is sporadic, or as extinct cometary nuclei whose activity has already stopped. Many attempts have been made to detect the faint coma of this object (Cochran \& Barker 1984; Chamberlin et al. 1996; Hsieh $\&$ Jewitt 2005). No cometary activity has been observed yet; therefore, (3200) Phaethon seems to be one of the most peculiar and enigmatic objects in the solar system.

On October 22, 2005, an Apollo-type, near-Earth asteroid was discovered by the Catalina Sky Survey, and the provisional designation of 2005 UD was given to this object (McNaught et al. 2005). Ohtsuka et al. (2005) immediately pointed out that 2005 UD was probably the parent body of the daytime Sextantids meteor stream, based on the orbital similarity. Thus, 2005 UD is very likely a large member of the Phaethon-Geminid stream complex. Furthermore, Ohtsuka et al. (2006) performed both forward and backward $10000-y r$ numerical integrations of the Kustaanheimo-Stiefel (K-S) regularized equation of motion
(Arakida \& Fukushima 2000, 2001) for (3200) Phaethon and 2005 UD. The results of this numerical work show quite similar evolutional behaviors along with the time shift by 4600-yr; hence, they suggest that both bodies are dynamically related and 2005 UD is most likely a km-sized fragment of (3200) Phaethon. Ohtsuka et al. (2006) also point out that fragmentation processes are important in forming the complex meteor stream.

Very recently, Jewitt \& Hsieh (2006) have reported their analysis of the photometric observation of 2005 UD. The results of their broadband multi-color photometry support the possible dynamical association between 2005 UD and (3200) Phaethon. The mass-loss rate from 2005 UD, based on the point-spread function (PSF) fitting, is found to be very small or negligible. They also mention that a mainbelt comet (MBC) could be a possible origin of (3200) Phaethon and 2005 UD, although testing their idea currently does not seem to be easy.

We carried out observational studies of 2005 UD. The primary aim of our observations was to verify the dynamical relationship between (3200) Phaethon and 2005 UD through the similarity of surface physical properties. If 2005 UD exhibited photometric properties similar to those of (3200) Phaethon, we can confirm prior research by providing another strong piece of evidence for their genetic relationship. Second, we tried to detect the surface color variation of 2005 UD. Ohtsuka et al. (2006) concluded 2005 UD is very likely a km-order fragment from (3200) Phaethon from the standpoint of dynamics; therefore, 
there may be non-uniformity of photometric properties if the fresh materials are exposed elsewhere on the surface. This sort of exposed fresh materials may create color variations during the rotation of the body.

We briefly introduce our observations, data analysis procedures, and results in Sect. 2. We give the discussion in Sect. 3 and summarize our results in Sect. 4.

\section{Observation, analysis, and results}

Photometric observations of 2005 UD were carried out at Lulin Observatory operated by the Institute of Astronomy, National Central University in Taiwan. The Lulin Observatory sits on the peak of Mount Lulin $\left(120^{\circ} 52^{\prime} 25^{\prime \prime} \mathrm{E}, 23^{\circ} 28^{\prime} 7^{\prime \prime} \mathrm{N}, H=2862-\mathrm{m}\right)$ in the central region of Taiwan. We used the Princeton Inc. CCD (charge coupled device) camera, PI1300B, with the Cassegrain focus $(\mathrm{F} / 8.0)$ of the $1-\mathrm{m}$ telescope to obtain imaging data. The pixel scale of this system is 0.516 arcsec per pixel, and it is spatially well-sampled at the typical seeing size of stellar FWHM (full width at half maximum), $\sim 1.5$ arcsec. The number of pixels of the CCD is $1340 \times 1300$, and the resultant field-of-view is $11.5 \times 11.2 \mathrm{arcmin}$. The cooling temperature of the CCD is set to $-50{ }^{\circ} \mathrm{C}$, and this is achieved by both thermoelectric cooling and water circulation. The filter set is based on the Bessel system. More detailed specifications and performances of the instrument have been reported by Kinoshita et al. (2005).

The data was acquired on six nights from 31 October to 5 November 2005. At the time of observations, the heliocentric distance of the object ranged from 1.36 to $1.42 \mathrm{AU}$, and its geocentric distance ranged from 0.51 to $0.62 \mathrm{AU}$. The apparent motion of 2005 UD is relatively large and non-sidereal tracking was used for this target. We used BVRI filters to measure the surface colors of 2005 UD for taxonomic study and color variation. To minimize the rotational effect, observations were done in $R$ $B-R-V-R-I-R$ sequences. This sequence of observation at least secured the rotational lightcurve when the sky condition was unstable. The exposure time was set to $180 \mathrm{~s}$ for the $R$-band, and $300 \mathrm{~s}$ for other bands. When the sky was photometric, standard stars from the list provided by Landolt (1992) were observed during the night. Photometric standard stars were carefully selected to cover wide ranges of airmass and colors for accurate correction of atmospheric and instrumental signatures. The number of Landolt fields we obtain was typically 20 per night, and this gave about 50-70 measurements of photometric standards. The flatfield frames were obtained using the twilight sky, both in the evening and morning.

The dark subtracting and flatfielding were applied in the standard manner using an image-analysis software package NOAO IRAF. Then, plate constants were refined by WCSTools (Mink 2002) for astrometric measurements. By including our 183 astrometric positions and the newly found precovery positions in 1982 and 2001, hence three oppositions until now, Nakano (2005) improved the orbit of 2005 UD.

\subsection{Differential photometry and rotational lightcurve}

Differential photometry was performed using $R$-band images. We used Lomb's algorithm for unevenly sampled data (Press et al. 1992) to extract the periodicity from relative magnitudes of 2005 UD. Ten field stars are selected as references. To combine measurements from different nights, we corrected the effect of the heliocentric and geocentric distance of the target. The change in the phase angle is about 0.2 degree during our observations, so was ignored. The Lomb periodogram for 2005 UD

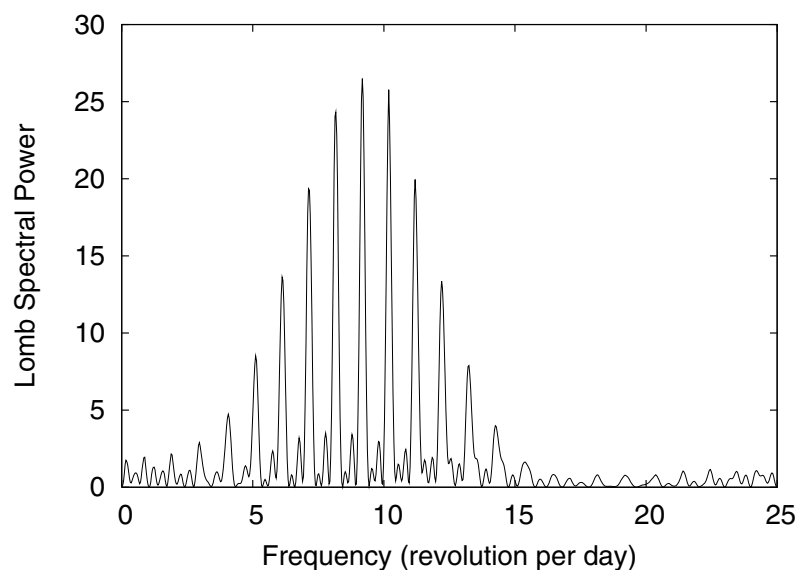

Fig. 1. The Lomb periodogram of 2005 UD. The highest peak corresponds to the period of $2.6155 \mathrm{~h}$.

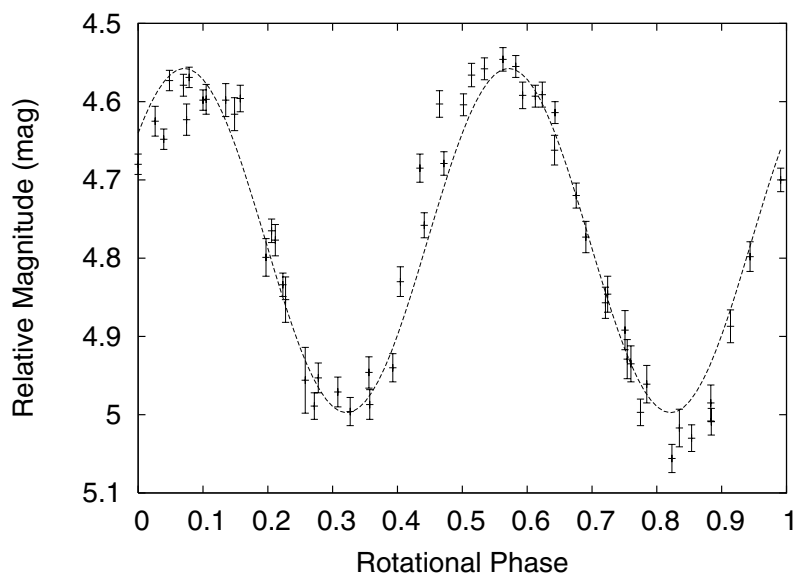

Fig. 2. The rotational phase curve of 2005 UD assuming periodicity of $5.2310 \mathrm{~h}$ is shown. The dotted curve is the simple sinusoidal fit to the data. The fitted amplitude is $0.45 \pm 0.02 \mathrm{mag}$.

is shown in Fig. 1. The periodogram exhibits the maximum at a period of $2.6155 \mathrm{~h}$. Considering the lightcurve is due to the rotation of the irregularly shaped body, double the obtained periodicity $5.2310 \mathrm{~h}$ is the actual rotational period. Figure 2 is the phase curve assuming the periodicity of 5.2310 h. Jewitt \& Hsieh (2006) utilized the phase dispersion minimization (PDM) method to obtain the rotational period of $5.2492 \mathrm{~h}$, which is quite close to ours.

The lower limit of the axis ratio of 2005 UD is calculated from the amplitude of the lightcurve, assuming that the variability comes entirely from the elongated shape of the object. The amplitude of the lightcurve is $0.44 \pm 0.02 \mathrm{mag}$, which implies the lower limit of the axis ratio of 1.50 from the relation

$\frac{a}{b}>10^{0.4 \Delta m}$

where $a / b$ is the axis ratio of primary and secondary axes, and $\Delta m$ is the amplitude of the lightcurve. Assuming 2005 UD has a weakly connected rubble-pile structure, we can set the lower limit of the bulk density (Jewitt \& Sheppard 2002; Ortiz et al. 2006). Giving the simple shape model of a Jacobi ellipsoid with an axis ratio of $a / b=1.50$, and the rotation period of $5.2310 \mathrm{~h}$, the critical density of 2005 UD is estimated as $1.5 \times 10^{3} \mathrm{~kg} \mathrm{~m}^{-3}$ by referring to the table in Chandrasekhar (1969). This lower limit implies an asteroidal picture for the bulk properties of 
Table 1. The transformation coefficients on 3, 4, 5, November 2005.

\begin{tabular}{lrrr}
\hline \hline & 03/Nov./2005 & 04/Nov./2005 & 05/Nov./2005 \\
\hline$Z_{B}$ & $+22.77 \pm 0.03$ & $22.85 \pm 0.01$ & $22.83 \pm 0.01$ \\
$k_{B}$ & $0.15 \pm 0.02$ & $0.18 \pm 0.01$ & $0.17 \pm 0.01$ \\
$C_{B}$ & $+0.12 \pm 0.01$ & $+0.15 \pm 0.01$ & $+0.14 \pm 0.01$ \\
$Z_{V}$ & $+23.08 \pm 0.03$ & $23.14 \pm 0.01$ & $23.11 \pm 0.01$ \\
$k_{V}$ & $0.08 \pm 0.02$ & $0.10 \pm 0.01$ & $0.09 \pm 0.01$ \\
$C_{V}$ & $-0.09 \pm 0.01$ & $-0.07 \pm 0.01$ & $-0.06 \pm 0.01$ \\
$Z_{R}$ & $+23.04 \pm 0.03$ & $23.09 \pm 0.01$ & $23.05 \pm 0.01$ \\
$k_{R}$ & $0.06 \pm 0.02$ & $0.07 \pm 0.01$ & $0.08 \pm 0.01$ \\
$C_{R}$ & $-0.13 \pm 0.02$ & $-0.11 \pm 0.01$ & $-0.08 \pm 0.01$ \\
$Z_{I}$ & $+22.37 \pm 0.03$ & $22.38 \pm 0.01$ & $22.36 \pm 0.02$ \\
$k_{I}$ & $0.05 \pm 0.02$ & $0.04 \pm 0.01$ & $0.03 \pm 0.01$ \\
$C_{I}$ & $+0.02 \pm 0.01$ & $+0.04 \pm 0.01$ & $+0.04 \pm 0.01$ \\
\hline & & &
\end{tabular}

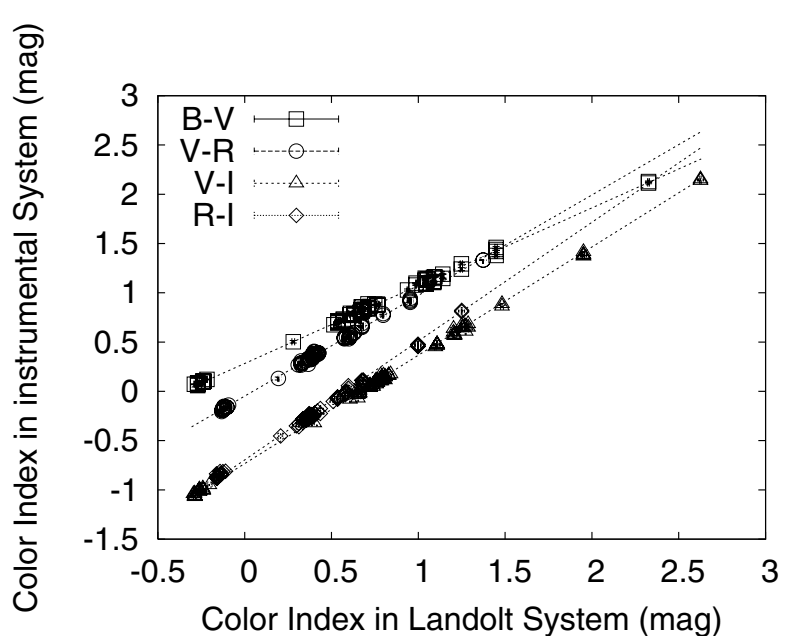

Fig. 3. Landolt colors and the atmospheric-extinction-corrected instrumental colors of Landolt photometric standards are plotted for $(B-V)$, $(V-R),(V-I),(R-I)$. The dotted straight lines are the least-square fit to the data. The data were obtained on November 3, 2005.

2005 UD, rather than the cometary picture. The amplitude of the lightcurve, axis ratio, and critical density agrees well with values reported by Jewitt \& Hsieh (2006).

\subsection{Multi-color photometry}

The multi-color photometry was performed with $B, V, R$, and $I$ filters. The transformation coefficients were estimated using the "photcal" package of NOAO IRAF. We defined transformation equations as

$B_{\text {std }}=B_{\text {inst }}+Z_{B}-k_{B}^{\prime} X+C_{B}(B-V)$,

$V_{\text {std }}=V_{\text {inst }}+Z_{V}-k_{V}^{\prime} X+C_{V}(B-V)$,

$R_{\text {std }}=R_{\text {inst }}+Z_{R}-k_{R}^{\prime} X+C_{R}(V-R)$,

$I_{\text {std }}=I_{\text {inst }}+Z_{I}-k_{I}^{\prime} X+C_{I}(V-I)$,

where $B_{\text {std }}, V_{\text {std }}, R_{\text {std }}, I_{\text {std }}$ are the standard magnitudes, $B_{\text {inst }}, V_{\text {inst }}$, $R_{\text {inst }}, I_{\text {inst }}$ are the instrumental magnitudes, $Z_{B}, Z_{V}, Z_{R}, Z_{I}$ are photometric zero points, $k_{B}^{\prime}, k_{V}^{\prime}, k_{R}^{\prime}, k_{I}^{\prime}$ are the first-order extinction coefficients, $C_{B}, C_{V}, C_{R}, C_{I}$ are the color terms, and $X$ is the airmass. The second-order extinction coefficients are found to be small on clear nights from our previous observations, so are neglected for this study. The transformation coefficients obtained on November 3, 4, and 5, 2005 are summarized in Table 1. These values are in good agreement with those reported by Kinoshita et al. (2005). The color conversions between the instrumental
Table 2. The coefficients of conversions between the Landolt colors and the instrumental colors, where the numbers in parentheses are errors of the fit.

\begin{tabular}{lrrr}
\hline & & & \\
\hline \hline$c_{B-V}$ & $1.270(0.006)$ & $1.264(0.005)$ & $1.266(0.004)$ \\
$z_{B-V}$ & $-0.366(0.005)$ & $-0.359(0.004)$ & $-0.357(0.003)$ \\
$c_{V-R}$ & $0.980(0.005)$ & $0.980(0.004)$ & $0.978(0.004)$ \\
$z_{V-R}$ & $0.047(0.002)$ & $0.049(0.002)$ & $0.048(0.002)$ \\
$c_{V-I}$ & $0.920(0.003)$ & $0.906(0.002)$ & $0.909(0.003)$ \\
$z_{V-I}$ & $0.675(0.002)$ & $0.676(0.002)$ & $0.667(0.002)$ \\
$c_{R-I}$ & $0.836(0.005)$ & $0.823(0.005)$ & $0.828(0.004)$ \\
$z_{R-I}$ & $0.591(0.002)$ & $0.584(0.002)$ & $0.577(0.002)$ \\
\hline
\end{tabular}

Table 3. The mean surface colors of 2005 UD.

\begin{tabular}{lcccl}
\hline \hline & $B-V$ & $V-R$ & $R-I$ & Ref. \\
\hline 2005 UD & $0.63 \pm 0.01$ & $0.34 \pm 0.01$ & $0.30 \pm 0.01$ & This work \\
2005 UD & $0.66 \pm 0.02$ & $0.35 \pm 0.02$ & $0.33 \pm 0.02$ & Jewitt et al. (2006) \\
(3200) Phaethon & - & 0.34 & - & Skiff et al. (1996) \\
(3200) Phaethon & $0.59 \pm 0.01$ & $0.35 \pm 0.01$ & $0.32 \pm 0.01$ & Dundon (2005) \\
\hline
\end{tabular}

system and the Landolt system are also performed. Figure 3 shows an example result of the color-color conversion. The data points are fitted by straight lines. We used the following formulae,

$$
\begin{aligned}
(B-V) & =c_{B-V} \times(b-v)+z_{B-V}, \\
(V-R) & =c_{V-R} \times(v-r)+z_{V-R}, \\
(V-I) & =c_{V-I} \times(v-i)+z_{V-I}, \\
(R-I) & =c_{R-I} \times(r-i)+z_{R-I},
\end{aligned}
$$

where capital letters refer to the Landolt system and lower-case letters refer to the instrumental system. The coefficients $c_{B-V}$, $c_{V-R}, c_{V-I}, c_{R-I}, z_{B-V}, z_{V-R}, z_{V-I}$, and $z_{R-I}$ are constants, and they are listed in Table 2 . These values are also consistent with those reported by Kinoshita et al. (2005).

Using the results of the photometric calibration shown above, we derived surface colors of 2005 UD. The photometry was done using a circular aperture three times larger than FWHM. After the atmospheric extinction correction, we scaled individual $B V R I$ photometric measurements to the average brightness, using the rotational phase. Then, instrumental colors were converted into the standard colors. The mean colors of 2005 UD averaged over the entire rotational lightcurve are summarized in Table 3. These mean colors are plotted in the $(B-V, V-R)$ space, together with the typical colors of major sub-groups of asteroids in Fig. 4. Similar to those of asteroids with primordial characteristics (cf. C-type and its subclasses), 2005 UD exhibits a bluish surface. From the broadband photometry, 2005 UD is most likely an F-type or B-type asteroid. Our measurements of the mean colors of 2005 UD agree with those of (3200) Phaethon (Skiff et al. 1996; Dundon 2005). This similarity in surface colors between 2005 UD and (3200) Phaethon supports the hypothesis that 2005 UD is a fragment of (3200) Phaethon. Recently, Jewitt \& Hsieh (2006) has also reported the photometric measurements of 2005 UD. Their measurements of the mean colors of 2005 UD are consistent with ours.

Next, we checked the color variation during the rotation of 2005 UD. Figures 5-7 show the $(B-V),(V-R),(R-I)$ colors of $2005 \mathrm{UD}$ at different rotational phases. In addition to our own measurements, results from Jewitt \& Hsieh (2006) are also superimposed on the figures. In all three figures, there is no inconsistency between this work and Jewitt \& Hsieh (2006). The 


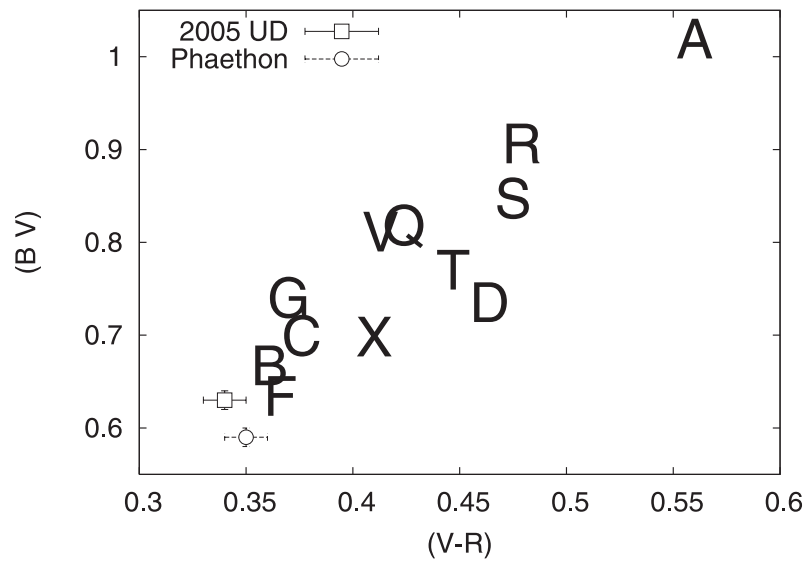

Fig. 4. The mean surface colors of 2005 UD from this work in the $(B-V$, $V-R)$ two-color diagram using open squares. The result of surface color measurements of (3200) Phaethon from Dundon (2005) is also superimposed in the figure, using open circles. The capital letters denote typical colors of major sub-groups of asteroids. These values are from Dandy et al. (2003).

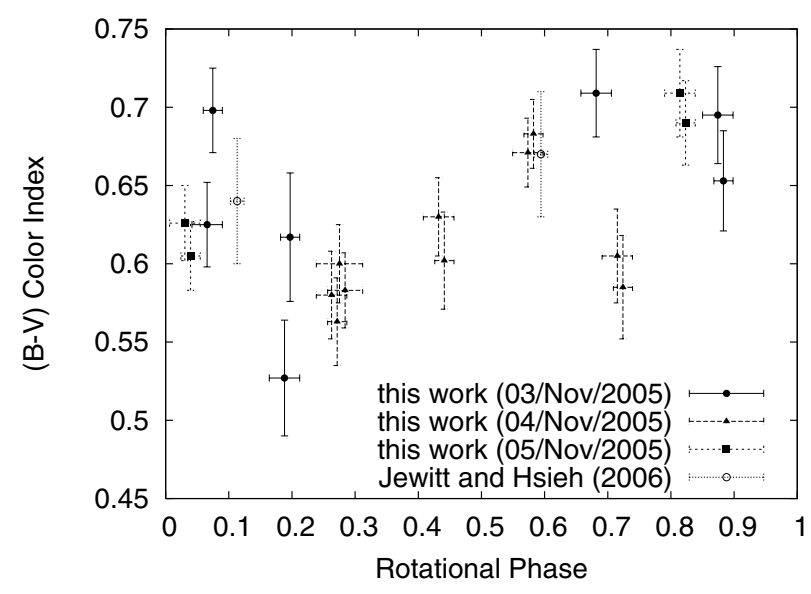

Fig. 5. The $(B-V)$ color of 2005 UD. The horizontal axis is the rotational phase. In addition to our own measurements, the results from Jewitt \& Hsieh (2006) are also superimposed on the figure.

$(R-I)$ color is not uniform, and the color variation is recognized in Fig. 7, where it exhibits bluer at the rotational phase of 0.9 0.3 . It is not as clear as $(R-I)$, but possible variation is seen also in $(B-V)$ in Fig. 5. No clear trend is identified for $(V-R)$. Figure 8 shows the scaled reflectance of 2005 UD normalized at the $V$-band. The rotational phase is divided into four regions $(0.20-0.45,0.45-0.70,0.70-0.95,0.95-0.20)$, and reflectances are shown for each of the rotational phase regions. These four regions correspond to the first maximum, first minimum, second maximum, and the second minimum of the lightcurve. For the rotational phase between 0.45 and 0.70 , the spectra of 2005 UD are as neutral as these of the Sun (Fig. 8 B). At other rotational phase regions, a slight decrease in reflectance is found at the $I$ band (Figs. 8A, C, D).

\section{Discussion}

The surface color of 2005 UD differs at different rotational phases. Such color variations are also found for (832) Karin, the largest member of the Karin family (Sasaki et al. 2004; Yoshida et al. 2004). The surface color heterogeneity does not seem to be rare for relatively young fragments. In the first-order

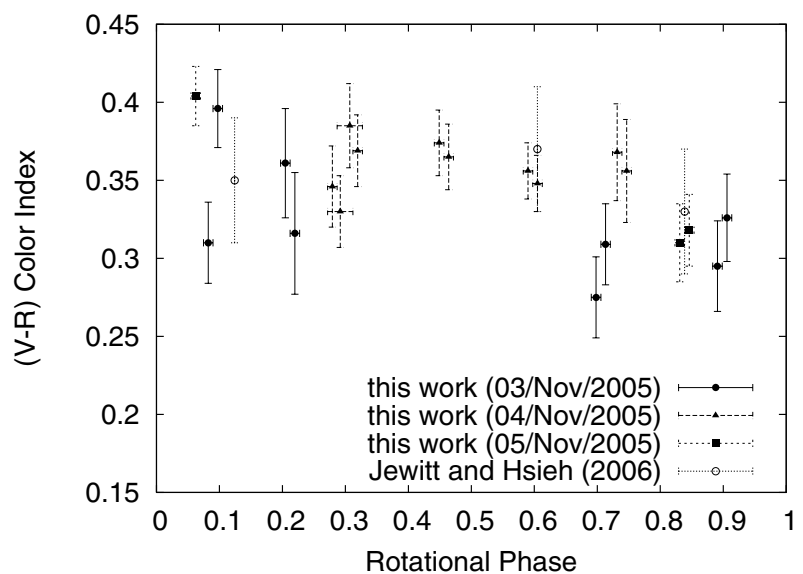

Fig. 6. Same as Figure 5, but for $(V-R)$.

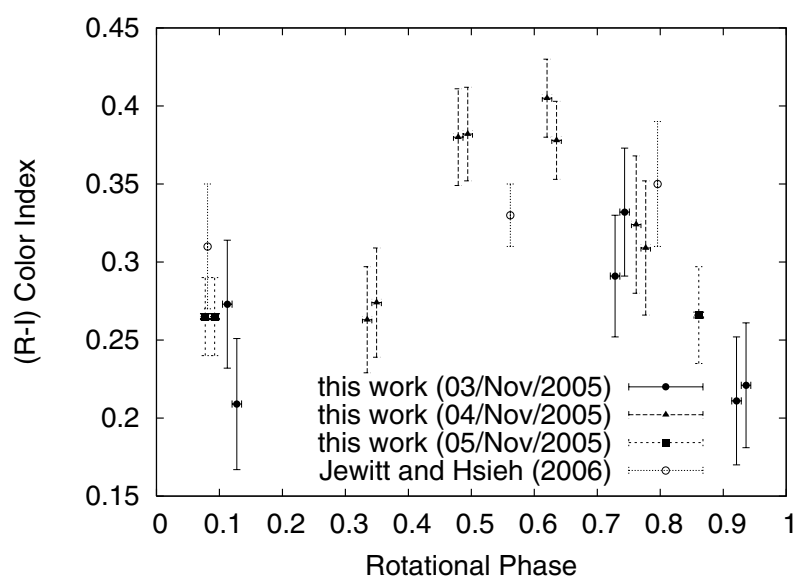

Fig. 7. Same as Fig. 5, but for $(R-I)$.

approximation, the overall spectral behavior of 2005 UD is consistent with low-albedo carbonaceous asteroids.

We divide the surface of 2005 UD into two distinct areas. One is the surface of a C-type asteroid analogue with flat reflectance of mostly solar colors. The rotational phase between 0.45 and 0.70 corresponds to this area. The other is the surface of an F-type asteroid analogue with slightly bluer reflectance compared to the solar colors. The area other than the C-type analogue surface corresponds to this area. In current understanding, thermal metamorphism turns C-type asteroid materials into F-type asteroid materials. The dehydration of hydrated minerals may be the key process in this change. The surfaces of F-type asteroids are thought to experience high temperature. The temperature is high enough to dehydrate hydrated minerals, and they form anhydrous minerals after thermal metamorphism (Rivkin et al. 2002). The C-type analogue area is most likely to consist of less thermally processed materials. The main issue is the coexistence of $\mathrm{C}$ - and F-type materials on a single asteroid. In this section, we discuss possible explanations for the surface heterogeneity of 2005 UD.

We assume the precursor object experienced fragmentation at some time in the past, after which the current (3200) Phaethon and 2005 UD were formed. If the split of the precursor object was due to the collision of two objects, part of 2005 UD may have come from the projectile. A simple explanation for the inhomogeneity of 2005 UD surface is that part of 2005 UD is from the precursor object and the rest is from the projectile. If the surface composition of the precursor object and the projectile are 

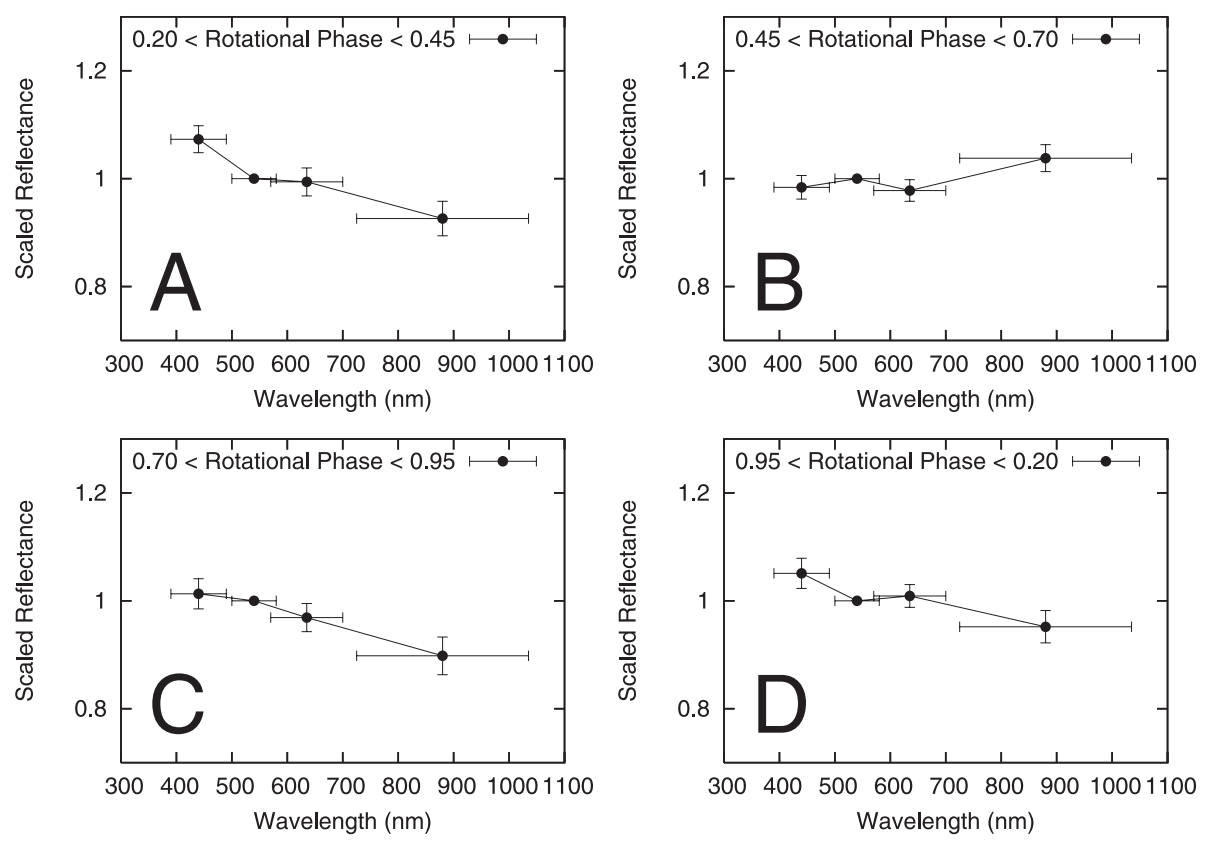

Fig. 8. Scaled reflectances normalized at the $V$-band of 2005 UD are shown. The rotational phase is divided into four regions $(0.20-0.45$, $0.45-0.70,0.70-0.95,0.95-0.20)$, and scaled reflectances are calculated for each rotational phase region. Panels A, B, C and D correspond to rotational phases of $0.20-0.45,0.45-0.70$, $0.70-0.95$, and $0.95-0.20$, respectively.

intrinsically different, the surface color variation of 2005 UD is easily understood. We call this the "precursor-projectile" model. The difficulty with this model is that the projectile must also be a bluish object, which is not a large population among near-Earth asteroids.

A second explanation is the combination of the alteration and resurfacing mechanism on the surface. It is widely accepted that the thermal alteration (or metamorphism) and space-weathering gradually changes the surface colors with time (ex. Sasaki et al. 2001). For asteroids of C-type and its subclasses, the thermal alteration makes the surface bluer (Hiroi et al. 1993). In addition, seismic shaking by impact or tidal distortion by planetary encounter may be mechanisms of resurfacing on $1-\mathrm{km}$ or smaller sized asteroids (Saito et al. 2006), and these processes remove some portions of the altered surface layer. This resurfacing mechanism exposes the fresh materials on the surface. We call this the "alteration-resurfacing" model. The difficulty with this model is that the patchy surface may not be detected from the ground by disk-integrated photometry.

A third explanation is that we see the surface and subsurface of the precursor object on the surface of the current 2005 UD. The thermal history of the surface and subsurface of the precursor object must be different, and the current 2005 UD exhibits different surface properties on different sides. We call this the "surface-subsurface" model. A part of the surface on 2005 UD that originated on the surface of the precursor object is likely to be covered with more anhydrous mineral. Another part of the surface that originated in the subsurface of the precursor object is probably covered with more hydrated mineral. The important factor for this model is the timescale of the alteration. If the timescale of the alteration is short enough compared to the time elapsed from the fragmentation of the precursor, no difference may be detected in the degree of alteration. Thus, this model requires that the age of 2005 UD be relatively young.

The last explanation is based on the topographical structure on the surface. A number of spacecraft explorations have revealed the existence of large craters on the surfaces of asteroids. Asteroids can support craters with sizes comparable to their diameters (Thomas 1999). The surface of an asteroid is excavated when a crater is formed so one can expect fresh materials to be exposed in the crater. We call this the "crateringexposition" model. Similar to the discussion for the surfacesubsurface model, the age of the crater must be less than the timescale of the alteration of the surface. However, the formation time of 2005 UD is less restricted for this model. The age of 2005 UD can be as old as the typical dynamical lifetime of near-Earth asteroids.

We believe that either the surface-subsurface or crateringexposition model is more plausible to explain the surface heterogeneity of 2005 UD. To test these ideas, a combination of more observations, theoretical work, and laboratory experiments are essential. For observational study, spectroscopic monitoring covering the entire rotational phase is strongly encouraged. Timeresolved polarimetric measurements are also effective.

\section{Summary}

An Apollo-type near-Earth asteroid 2005 UD has been considered to be associated with the daytime Sextantids meteor stream and to be a fragment of (3200) Phaethon. To test this hypothesis, we performed photometric observations of 2005 UD using the 1-m telescope at Lulin Observatory in Taiwan to test this hypothesis. The time-resolved photometry in the $R$-band showed a rotational period of $5.2310 \mathrm{~h}$. The lower limit of the axis ratio $a / b \sim 1.50$ is estimated from the amplitude of the lightcurve. The lower limit of the bulk density of 2005 UD is estimated as $1.5 \times 10^{3} \mathrm{~kg} / \mathrm{m}^{3}$. Results of multi-color photometry show a bluish surface for 2005 UD, which is consistent with (3200) Phaethon, and this supports the hypothesis that 2005 UD is a kmsized fragment of (3200) Phaethon. Furthermore, surface colors of 2005 UD exhibit variations during the rotation of the body. This surface inhomogeneity may be associated with the fragmentation or collisional processes.

Acknowledgements. We would like to express our hearty thanks to Mr. JunShiung Shih, Mr. Jing-Chuan Du, Mr. Hao-Wei Shih, and Mr. Zong-Jing Wan for their local support at Lulin Observatory. One of the authors (KD) thanks the fellowship from the Japan Society for Promotion of Science (ID: 15-1-202681-1) that provided partial support for this study. This work was also supported in part by the Ministry of Education of Taiwan under the "Aim for Top University Program". Authors also would like to thank Dr. Tatsuaki Okada, Prof. Sho Sasaki, and Dr. Masanao Abe for fruitful discussions at the 3rd annual meeting of AOGS in Singapore and 36th COSPAR scientific assembly in Beijing. 


\section{References}

Arakida, H., \& Fukushima, T. 2000, AJ, 120, 3333

Arakida, H., \& Fukushima, T. 2001, AJ, 121, 1764

Chamberlin, A. B., McFadden, L.-A., Schulz, R., Schleicher, D. G., \& Bus, S. J. 1996, Icarus, 119, 173

Chandrasekhar, S. 1969, in Ellipsoidal Figures of Equilibrium (Dover Publication)

Cochran, A. L., \& Berker, E. S. 1984, Icarus, 59, 296

Dundon, L. 2005, master thesis at University of Hawaii

Dandy, C. L., Fitzsimmons, A., \& Collander-Brown, S. J. 2003, Icarus, 163, 363

Green, S., \& Kowal, C. 1983, IAU Circular, 3878

Gustafson, B. A. S. 1989, A\&A, 225, 533

Hiroi, T., Pieters, C. M., Zolensky, M. E., \& Lipschutz, M. 1993, Science, 261, 1016.

Hsieh, H. H., \& Jewitt, D. 2005, ApJ, 624, 1093

Jewitt, D., \& Hsieh, H. 2006, AJ, 132, 1624

Jewitt, D., \& Sheppard, S. 2002, AJ, 123, 2110

Kinoshita, D., Chen, C.-W., Lin, H.-C., et al. 2005, ChJAA, 5, 315

Landolt, A. U. 1992, AJ, 104, 340

McNaught, R. H., McGaha, J. E., Young, J., et al., Minor Planet Electronic Circ., 2005-U22
Mink, D. J. 2002, Proceedings of Astronomical Data Analysis Software and Systems XI, ASP Conf. Proc., 281, ed. D. A. Bohlender, D. Durand, \& T. H. Handley, 169

Nakano, S. 2005, http://www.spaceguard.or.jp/ja/mpnews/0036.html (in Japanese)

Ohtsuka, K., Sekiguchi, T., Kinoshita, D., Watanabe, J. 2005, Central Bureau Electronic Telegrams, 283

Ohtsuka, K., Sekiguchi, T., Kinoshita, D., et al. 2006, A\&A, 450, L25

Ortiz, J. L., Gutierrez, P. J., Santos-Sanz, P., Casanova, V., Sota, A. 2006, A\&A, 447,1131

Press, W., Flannery, B. P., Teukolsky, S. A., Vetterling, W. T. 1992, Numerical Recipes in C: The Art of Scientific Computing, (Cambridge University Press)

Rivkin, A. S., Howell, E. S., Vilas, F., Lebofsky, L. A. 2002, Asteroids III, (University of Arizona Press), 235

Saito, J, Miyamoto, H., Nakamura, R., et al. 2006, Science, 312, 1341

Sasaki, S., Nakamura, K., Hamabe, Y, Kurahashi, E., Hiroi, T. 2001, Nature, 410, 555

Sasaki, T., Sasaki, S., Watanabe, J., et al. 2004, ApJ, 615, L161

Skiff, B. A., Buie, M. W., Bowell, E. 1996, BAAS, 28, 1104

Thomas, P. C. 1999, Icarus, 142, 89

Whipple, F. L. 1983, IAU Circular, 3881

Williams, I. P., \& Wu, Z. 1993, MNRAS, 262, 231

Yoshida, F., Dermawan, B., Ito, T., et al. 2004, PASJ, 56, 1105 